\title{
Prevalence of Vitamin D Insufficiency in Severely Obese Patients
}

\section{Seeking Bariatric Surgery}

R. Smith ${ }^{1,2}$, R. Batterham ${ }^{1,3}$, N. Finer ${ }^{3,4}$

${ }^{1}$ Department of Medicine, University College London; ${ }^{2}$ Department of Medicine, Brighton and Sussex Medical School; ${ }^{3}$ UCLH Centre for Weight Loss, Metabolic and Endocrine Surgery; ${ }^{4} \mathrm{UCL}$ Institute of Cardiovascular Science

\section{Introduction}

Vitamin D deficiency and insufficiency is common in obese individuals. The cause of this relationship is not apparent. The vast majority of vitamin $D$ is synthesised through skin exposure to sunlight, although a small proportion can additionally be obtained via diet.

Current theories for the insufficient vitamin D concentrations include

- Vitamin $D$ sequestration by adipose tissue

- Inadequate sunlight exposure

Inadequate diet content of vitamin D

\section{Aims:}

Determine the prevalence of vitamin D insufficiency

within a cohort of severely obese individuals

Explore underlying associations

\section{Methods}

In a retrospective review of 703 consecutive patients presenting for bariatric surgery assessment, 663 were evaluated after excluding those with missing data.

\section{Assessment included}

- Clinical history: comorbidities, current medications, multivitamin use, smoking, alcohol

- Basic anthropometry: weight and height used to calculate indirect measures of adiposity; body mass index (BMI) and percentage body fat via the CUN-BAE equation

- Blood tests: haemoglobin $(\mathrm{Hb})$, serum iron, total iron binding capacity, iron binding saturation, ferritin, vitamin $B_{1}$, vitamin $B_{12}$, folate, white cell count (WCC), and Creactive protein (CRP)

Vitamin D status was classified according to local laboratory ranges:

- Normal: $>50 \mathrm{nmol} / \mathrm{L}$

- Insufficient: 25-50nmol/L

Deficient: $<25 \mathrm{nmol} / \mathrm{L}$

\section{Results}

Mean serum vitamin D concentration was $38 \pm 23 \mathrm{nmol} / \mathrm{L}$, much lower than levels observed in the normal population (men; $45.6 \mathrm{nmol} / \mathrm{L}$ vs women; $49.6 \mathrm{nmol} / \mathrm{L}$ ). ${ }^{1} \mathrm{No}$ difference in vitamin $D$ concentrations between males and females was observed in this obese cohort.

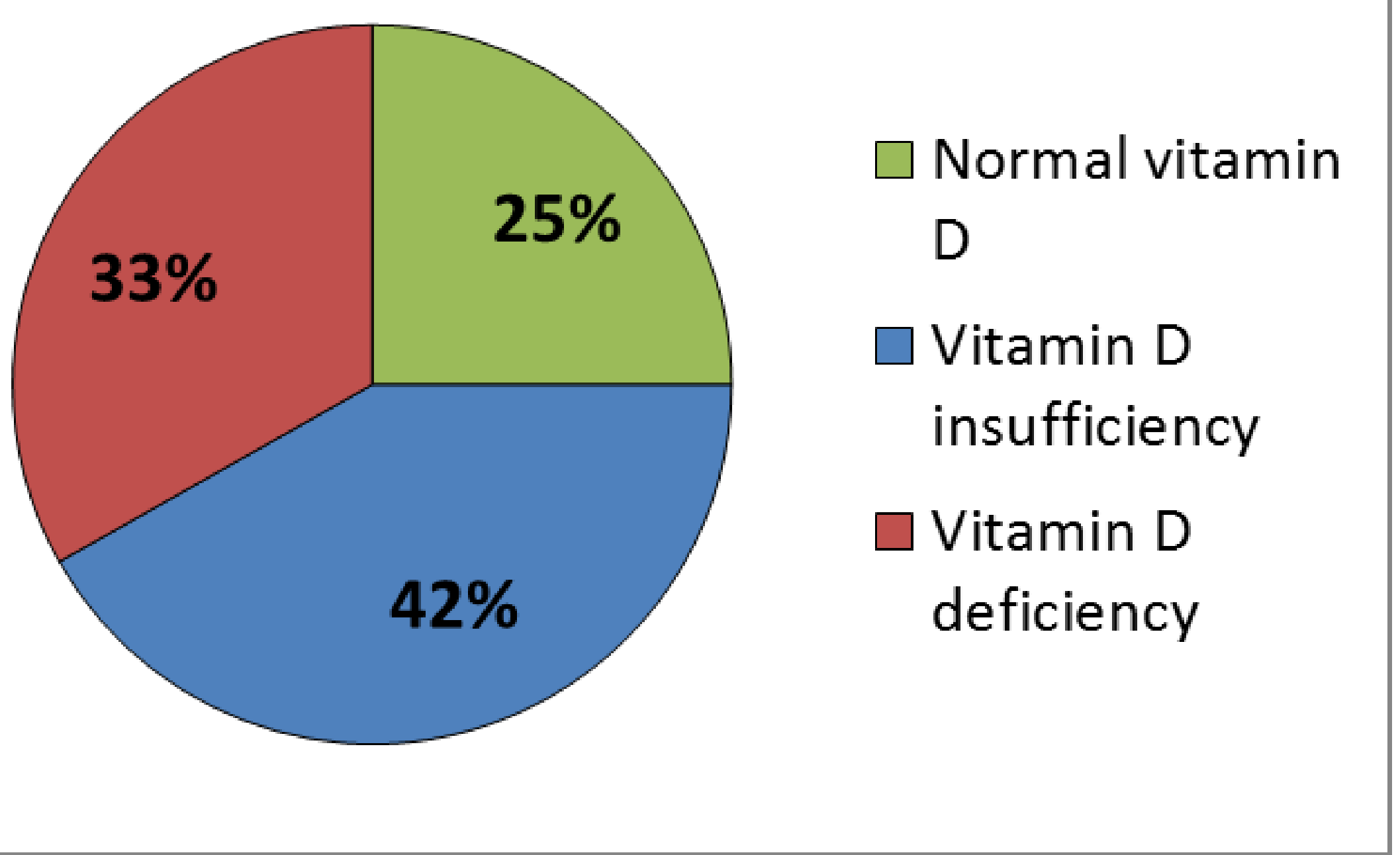

Figure One:

Proportions of patients falling into each of the

three categories of

vitamin D status;

normal, insufficient and deficient

The majority of patients had low vitamin D concentrations (75\%), and only $25 \%$ had normal concentrations. Cohort characteristics are presented in Table One.

Table One: Cohort characteristics according to vitamin D status classification. Values with the same superscript exhibit significant variation $(p<0.001)$.

\begin{tabular}{|llll|}
\hline & Normal & $\begin{array}{l}\text { Vitamin D } \\
\text { Insufficiency }\end{array}$ & $\begin{array}{l}\text { Vitamin D } \\
\text { Deficiency }\end{array}$ \\
\hline Age (years) & $46.7 \pm 12.0$ & $44.4 \pm 11.8$ & $44.3 \pm 12.8$ \\
\hline BMI (kg/m²) & $45.2 \pm 5.9^{\mathrm{a}}$ & $46.8 \pm 7.2^{\mathrm{a}}$ & $48.7 \pm 8.1^{\mathrm{a}}$ \\
\hline Body Fat Percentage & $52.8 \pm 4.7$ & $52.7 \pm 4.8$ & $53.4 \pm 4.8$ \\
\hline No. of comorbidities & $4 \pm 2$ & $4 \pm 5$ & $3 \pm 2$ \\
\hline
\end{tabular}

Table One shows that the groups of patients were very similar with no difference in age or number of comorbidities, however there was a significant difference in BMI. According to independent t-tests, those patients that had vitamin D deficiency had higher BMIs than both those with vitamin D insufficiency and those with normal vitamin D concentrations.
Although BMI was different between groups, calculated body fat percentage was not, suggesting that vitamin $\mathrm{D}$ concentration is not related to adiposity.

Vitamin D deficiency was also analysed examining relationships with other nutritional markers. These results are presented in Table Two.

Table Two: Nutritional markers according to vitamin $D$ status. Values with the same superscript exhibit significant variation $(p<0.05)$

\begin{tabular}{|llll|}
\hline & Normal & $\begin{array}{l}\text { Vitamin D } \\
\text { Insufficiency }\end{array}$ & $\begin{array}{l}\text { Vitamin D } \\
\text { Deficiency }\end{array}$ \\
\hline Iron (umol/L) & $14.9 \pm 5.4$ & $14.2 \pm 5.6$ & $14.2 \pm 5.4$ \\
\hline Folate $(\mathrm{ng} / \mathrm{mL})$ & $8.7 \pm 4.5^{\mathrm{a}}$ & $7.7 \pm 4.2^{\mathrm{b}}$ & $5.8 \pm 2.6^{\mathrm{a}, \mathrm{b}}$ \\
\hline Vitamin $\mathrm{B}_{\mathbf{1 2}}(\mathrm{pg} / \mathrm{mL})$ & $464 \pm 252^{\mathrm{c}}$ & $445 \pm 224^{\mathrm{d}}$ & $393 \pm 164^{\mathrm{c}, \mathrm{d}}$ \\
\hline Vitamin $\mathrm{B}_{\mathbf{1}}(\mathrm{nmol} / \mathrm{L})$ & $174 \pm 40$ & $169 \pm 44$ & $169 \pm 39$ \\
\hline
\end{tabular}

The results presented in Table Two show that those patients with vitamin D deficiency have lower folate $(p<0.001)$ and vitamin $B_{12}$ concentrations ( $\left.p=0.003\right)$.

Stepwise regression was performed using age, gender, BMI, BF\%, index of multiple deprivation, multivitamin use, number of comorbidities, WCC, CRP, folate and vitamin $B_{12}$ as independent variables for vitamin $D$ concentration. This model revealed only a weak, but statistically significant association with folate concentration $\left(R^{2}=0.081 ; p-\right.$ 0.046)

As vitamin D is synthesised following exposure to sunlight and in UK populations, vitamin $D$ deficiency is related to season, we analysed the prevalence of vitamin $D$ deficiency according to season of assessment. The results are presented in Figure Two.

Figure Two:

Prevalence of vitamin

D deficiency

according to season

of bariatric surgery

assessment.

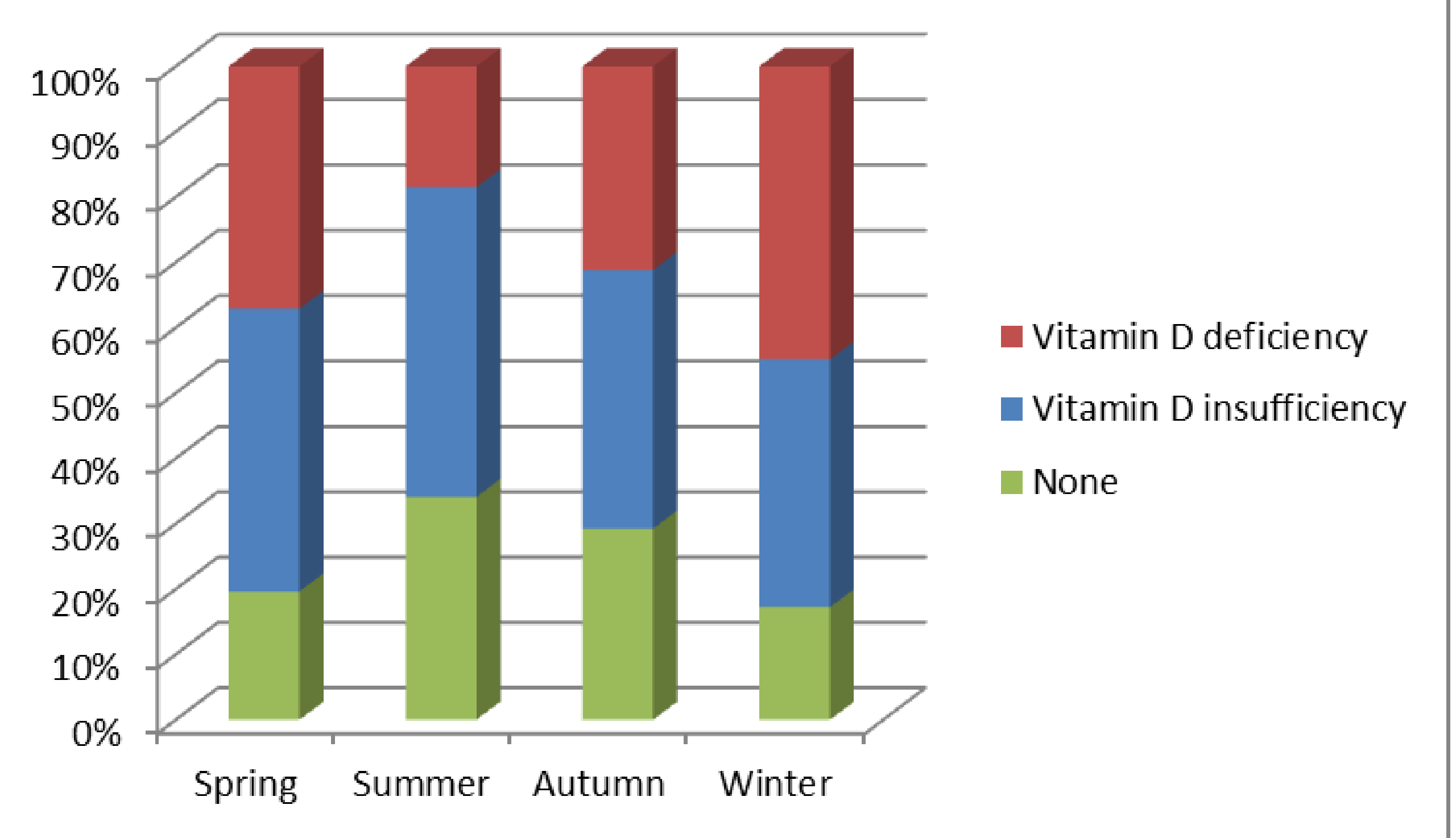

Chi-squared analysis proved a significant difference existed in rates of vitamin $D$ deficiency according to the season of assessment. However, the actual impact of this difference is minimal, as even in the summer at the height of vitamin D status, $70 \%$ of patients were still vitamin $D$ insufficient or deficient, although the mean vitamin $D$ concentration did approach that of the general population.

\section{Limitations}

- This was a retrospective, observational study so causality cannot be determined

- Although the cohort was large, some biochemical data was not available for all patients

- Body fat percentage was calculated via an equation and not measured directly, the equation used for this has not been accurately validated for this type of population

\section{Conclusions}

Vitamin D insufficiency is common among severely obese bariatric surgery candidates, observed in approximately $75 \%$ of patients. The underlying cause is still unknown and the hypotheses of sequestration within adipose tissue, or inadequate nutritional intake are not supported by these data. Questions remain regarding the functional significance of low vitamin D concentrations among obese individuals, and whether routine supplementation is required. 